\title{
Blood and Marrow Neutrophils during Experimental Group B Streptococcal Infection: Quantification of the Stem Cell, Proliferative, Storage and Circulating Pools
}

\author{
ROBERT D. CHRISTENSEN, ${ }^{(19)}$ JANE L. MACFARLANE, NANCY L. TAYLOR, HARRY R. HILL AND \\ GERALD ROTHSTEIN \\ Departments of Pediatrics, Medicine and Pathology, Primary Children's Medical Center and the University of Utah \\ College of Medicine, Salt Lake City, Utah, USA
}

\section{Summary}

In an attempt to explain the profound neutropenia and depletion of marrow neutrophil reserves observed in human and experimental group B streptococcal infection, we have studied the stem cell, proliferative, storage, and circulating neutrophil pools in groups of adult and neonatal rats inoculated with $3 \times 10^{6}$ type II group B streptococci/g body weight. At intervals after inoculation, animals were sacrificed and the unipotent granulocytic stem cells, (Colony Forming Units in culture, $\mathrm{CFU}_{c}$ ) from the femoral marrow were quantified. In addition, the total body neutrophil proliferative pool, neutrophil storage pool, circulating blood neutrophil concentration and blood immature to total neutrophil ratio were measured. Adult animals developed neutrophilia with a peak blood neutrophil concentration of 3.4 times control by $24 \mathrm{~h}$ after inoculation $(P<$
0.001). A left shift was seen in these mature animals as early as 2 $h$ but by $24 \mathrm{~h}$ was no longer present. The neutrophil storage pool diminished to $65 \%$ of control by $15 \mathrm{~h}(P<0.001)$ but then increased to exceed the control in all animals by $48 \mathrm{~h}(P<0.01)$. The neutrophil proliferative pool did not change during infection in the adult animals, but $\mathrm{CFU}_{\mathrm{c}}$ increased to three times control $48 \mathrm{~h}$ after inoculation $(P<0.001)$ and returned to baseline by $72 \mathrm{~h}$.

The myeloid response of the neonatal rats was markedly different from that of the adults. Neonates developed profound neutropenia with an extreme left shift, exhaustion of the neutrophil storage pool (less than $10 \%$ of control by $15 \mathrm{~h}, P<0.001$ ), and diminution of the proliferative pool to $58 \%$ of control by $10 \mathrm{~h}(P$ $<0.05$ ). In contrast to the adult animals, neonatal rats infected with group $B$ streptococci failed to increase $\mathrm{CFU}_{c}$, and in fact a diminution of $\mathrm{CFU}_{\mathrm{c}}$ to $48 \%$ of control was seen by $15 \mathrm{~h}(P<0.05)$.

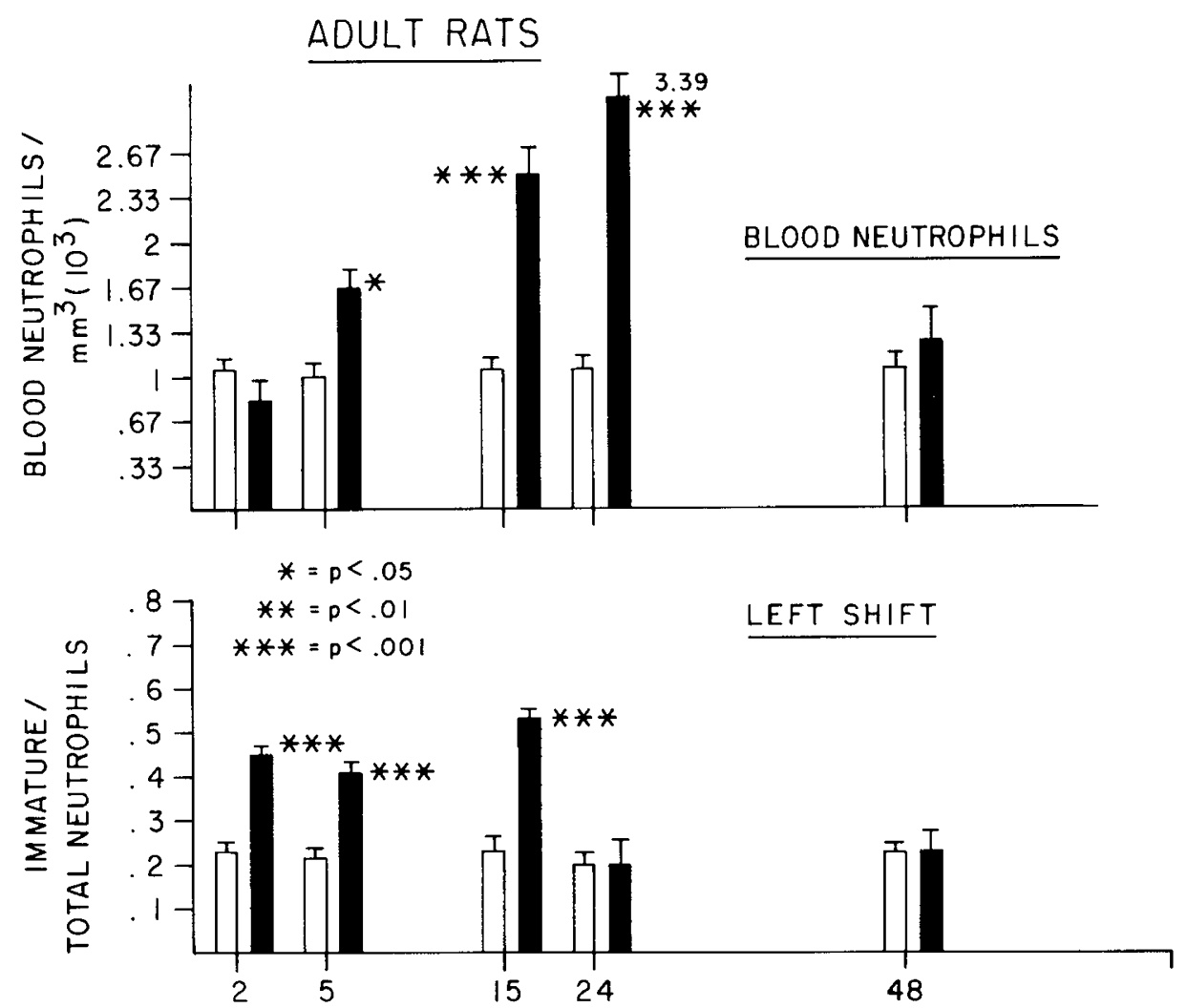

Fig. 1. Absolute blood neutrophil count and immature to total neutrophil ratio in groups of infected versus control adult rats. The bar indicates the mean of 5-8 animals and the bracket the S.E. 


\section{Speculation}

When adults develop bacterial infection, they often demonstrate neutrophilia and a leukocyte left shift. We observed these findings in infected adult rats accompanied by a moderate, temporary diminution of storage neutrophils and by an elevation in granulocytic stem cells. The adult response enables the animal to deliver large numbers of neutrophils to the infected tissues. In contrast, infected neonates developed profound neutropenia, exhaustion of neutrophil reserves and decreased neutrophil production. The neonatal response results in diminished delivery of neutrophils to infected tissues and a marked increase in mortality. The decreased production of neutrophils accompanying neutrophil storage pool depletion and neutropenia in neonates may constitute a condition for which neutrophil transfusion may represent a reasonable therapeutic approach.

Bacterial infection remains a major cause of mortality in the neonatal period (17). Experimental work suggests that this is largely due to a combination of neonatal host defense deficiencies (15). Among the deficiencies which have been reported, we have described the tendency of infected neonates to quickly exhaust their neutrophil reserves $(3,4)$. Neutrophil storage pool depletion results in blood neutropenia and a diminished supply of neutrophils with which to combat the infection (13). Depletion of the neutrophil storage pool during infection is rare in adults (14) but appears to be quite common in both animal and human neonates $(3,4,5)$. In the present study we explored this phenomenon by inoculating groups of newborn and adult rats with $3 \times 10^{6}$ group B streptococcus/g body weight. At intervals after the inoculation we measured the unipotent granulocytic stem cells $\left(\mathrm{CFU}_{\mathrm{c}}\right)$, proliferative, storage and circulating neutrophil pools in infected and control animals. Considerable differences in the myeloid tissue response to group $B$ streptococcal infection were observed between the neonatal and adult animals.

\section{MATERIALS AND METHODS}

Animal inoculation. Simonson Albino Sprague Dawley rats (Simonson Laboratories, Gilroy, CA) in the first $12 \mathrm{~h}$ of life, $(7 \pm 0.5$ g) or at 5-6 wk of age $(80 \pm 7 \mathrm{~g})$ were used. One microliter/g body weight of bacterial suspension was injected intraperitoneally with a glass syringe (Hamilton Co., Whittier, $\mathrm{CA}$ ) after preparing the abdominal wall by clipping the hair in the adult animals and washing the area with povidone-iodine (Perdue Frederick Co., Norwalk, CT) and 70\% ethyl alcohol. Control animals received 1 microliter/g body weight of sterile phosphate-buffered saline (PBS).

Type II Group B streptococci, which were isolated from an infected neonate, were identified by the precipitin method using rabbit antisera (11) and were grown overnight at $37^{\circ} \mathrm{C}$ in ToddHewitt broth. After washing in PBS, aliquots were frozen at $-70^{\circ} \mathrm{C}$; before inoculation into animals, strains were thawed and grown to logarithmic phase in fresh Todd-Hewitt broth. Bacteria were then sedimented by centrifugation and the concentrated organisms were washed 3 times in sterile PBS. A standard suspension was prepared by diluting the concentrated, washed organisms
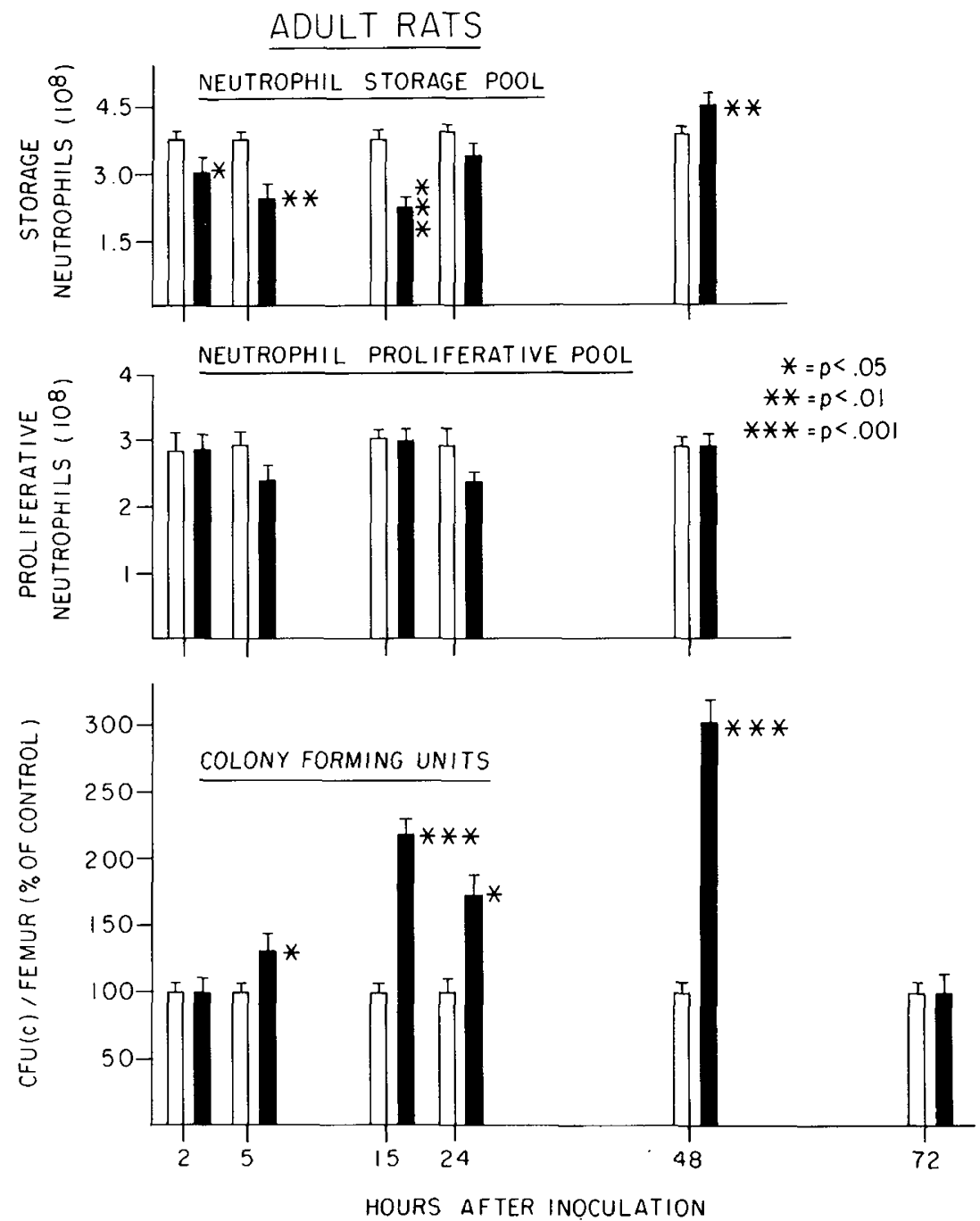

Fig. 2. Storage neutrophils, proliferative neutrophils and unipotent stem cells $\left(C F U_{c}\right)$ in groups of infected versus control adult rats. 
in PBS to an optical density of 0.9 at $620 \mathrm{~nm}$ (Spectronic 20, Bausch and Lomb, Inc., Rochester, NY) The suspensions contained approximately $3 \times 10^{6}$ colony-forming units/microliter.

Blood, peritoneal and pleural surface cultures from inoculated and control animals were plated onto sheep blood agar plates and were also grown in Todd-Hewitt broth.

$C F U_{c}$ quantification in agar culture. Bone marrow cells were flushed from the femurs of control and infected adult and neonatal rats into McCoy's 5A medium (Grand Island Biological Co., Grand Island, NY). Electronic cell counts (Coulter Electronics, Hialea FL) and coverslip smears were made on each cell suspension. Fifty thousand marrow cells were added to $1 \mathrm{ml} \mathrm{McCoy's}$ 5A with fetal calf serum, 3\% agar (Difco Laboratories, Detroit, MI), 200,000 units/liter penicillin $200 \mathrm{mg} /$ liter streptomycin, and $3 \%$ post endotoxin rat serum. The post endotoxin serum, the source of colony stimulating factor (18), was obtained from pooling the serum of ten adult rats $3 \mathrm{~h}$ after the intravenous injection of $10 \mu \mathrm{g}$ Salmonella typhosa endotoxin (Difco Laboratories, Detroit, MI) $/ \mathrm{g}$ body weight. The serum was frozen at $-70^{\circ} \mathrm{C}$ until use. The mixtures were plated in $10 \times 35 \mathrm{~mm}$ plastic petri dishes (Becton, Dickinson and Co., Oxnard, CA) and incubated at $37^{\circ} \mathrm{C}$ in a $7 \% \mathrm{CO}_{2}$, high humidity atmosphere (Wedco, Inc., Silver Springs, MD) for 7 days. Colonies of greater than 50 cells were counted using a stereomicroscope.

Quantification of proliferative, storage and circulating neutrophils. The neutrophil proliferative pool consisted of myeloblasts, promyelocytes, and myelocytes and the neutrophil storage pool the metamyelocytes, band neutrophils and PMN within the bone marrow, and in the neonatal rats, also within the spleen and liver. The hepatic and splenic compartments were measured after sacrificing the animal (cervical dislocation) by removing the liver and spleen and forcing the cells through a fine steel cloth mesh with $1.0 \mathrm{ml} \mathrm{Hanks-balanced} \mathrm{salt} \mathrm{solution.} \mathrm{The} \mathrm{resulting} \mathrm{cell} \mathrm{suspen-}$ sions, after being flushed twice through a $\# 25$ gauge needle, were counted electronically and a 1,000 cell differential count performed on Wright stained smears. The absolute nucleated cell count multiplied by the $\%$ of mytosable neutrophils was taken as the neutrophil proliferative pool. Similarly, the absolute nucleated cell count multiplied by the \% of nonmytosable neutrophils was taken as the neutrophil storage pool for these organs.

The bone marrow component of the proliferative and storage pools was measured as follows: the marrow content of both femurs was flushed into Hanks-balanced salt solution according to the method of Chervenick (2). The resulting cell suspension was counted electronically and a 1,000 cell differential count performed. The relationship of the femoral marrow to the total skeletal marrow was determined previously (6). Therefore the total body neutrophil proliferative pool and the total body neutrophil storage pool was calculated in individual rats by summing the absolute pool count of the animal's liver + spleen + the total skeletal pool.

\section{RESULTS}

Morbidity and mortality. All adult rats developed both local and systemic group B streptococcal infection. Cultures of the peritoneum were positive for up to five days while blood cultures were all positive at from 5-24 h. In addition, cultures of the pleural surface were positive in all adult animals $2 \mathrm{~h}$ following the intraperitoneal inoculation but became sterile by $5 \mathrm{~h}$. No adult animals died. Twenty-eight neonatal animals were inoculated with the same number of organisms/g body weight. They developed a lethal infection ( $\left(\mathrm{LD}_{90}\right)$ with death usually coming $18-20 \mathrm{~h}$ after inoculation. Post-mortem cultures of the blood, lung, liver and spleen were all positive for type II group B streptococci.

Blood and marrow neutrophil response in adult animals. The blood neutrophil count and the degree of left shift, (quantified as the immature/total neutrophil ratio) (5), in groups of five or more control and infected adult rats at intervals following inoculation is shown in Figure 1. Five $h$ after the inoculation, the infected animals had marked neutrophilia; with a blood neutrophil count of over three times control at $24 \mathrm{~h}$. By $48 \mathrm{~h}$ the blood neutrophil

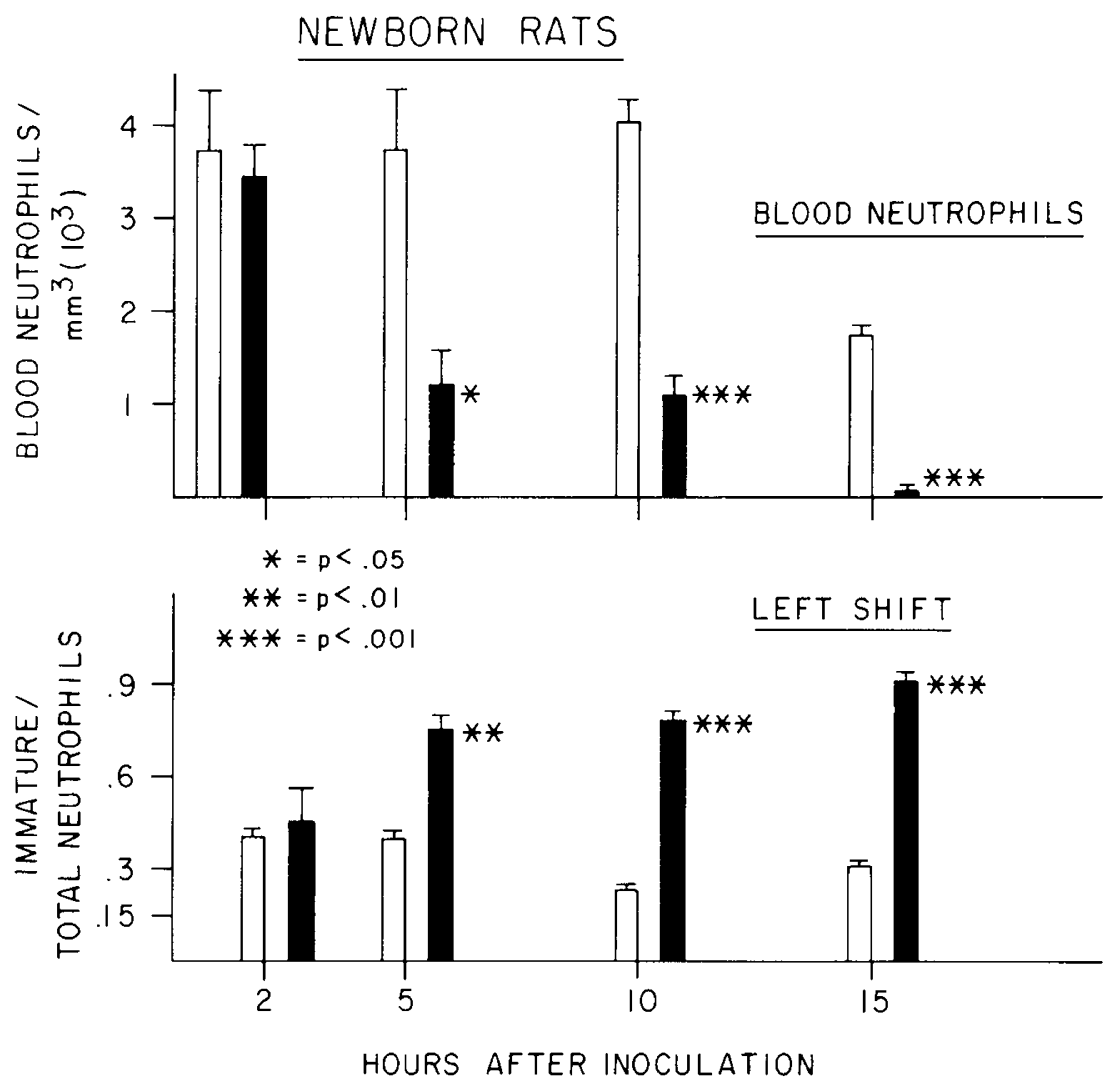

Fig. 3. Absolute blood neutrophil count and immature to total neutrophil ratio in groups of infected versus control neonatal rats. The bar indicates the mean of 5-8 animals and the bracket the S.E. 
count was back down within the range of the control animals. As shown, a left shift was apparent by $2 \mathrm{~h}$. The maximum $\mathrm{I} / \mathrm{T}$ ratio was at $15 \mathrm{~h}$ at which time over one-half of the circulating neutrophils were immature.

The neutrophil storage pool, neutrophil proliferative pool and the number of stem cells $\left(\mathrm{CFU}_{\mathrm{c}}\right)$ /femur in control and infected adult rats at intervals following the inoculation are shown in Figure 2. By $2 \mathrm{~h}$ following the inoculation, the storage neutrophils had diminished slightly from $3.7 \times 10^{8}$ cells to $3.0 \times 10^{8}(P<$ 0.05 ). Maximal diminution of the NSP occurred at $15 \mathrm{~h}$ in the infected animals at which time it equaled $60 \%$ of control. The neutrophil proliferative pool did not change appreciably during the course of illness. Stem cell number increased to over 3 times control values by $48 \mathrm{~h}$ and returned to within the control range by $72 \mathrm{~h}$.

Blood and marrow neutrophil response in neonatal animals. The blood neutrophil count and the blood left shift in groups of five or more control and infected neonatal animals at intervals after inoculation are shown in Figure 3. By $5 \mathrm{~h}$ animals were neutropenic and by $15 \mathrm{~h}$ all animals had fewer than 270 circulating neutrophils $/ \mathrm{mm}^{3}$. A left shift was evident $2 \mathrm{~h}$ after inoculation and was greater than 0.9 by $15 \mathrm{~h}$ indicating that over $90 \%$ of the circulating neutrophils were immature cells.

The neutrophil storage pool, neutrophil proliferative pool and the number of stem cells $\left(\mathrm{CFU}_{c}\right)$ /femur in control and infected neonatal rats at intervals following the inoculation are shown in
Figure 4 . By 5 h the storage pool was reduced to $30.8 \%$ of control $(P<0.001)$, and by $15 \mathrm{~h}$ contained only $1.1 \times 10^{6}$ cells or $9 \%$ of control $(P<0.001)$. The neutrophil proliferative pool did not differ from that of controls at 2 and $5 \mathrm{~h}$, but at 10 and $15 \mathrm{~h}$ a reduction was observed $(P<0.001)$. The $\mathrm{CFU}_{\mathrm{c}} /$ femur did not increase as occurred in the adult animals; in fact at $15 \mathrm{~h}$ after the inoculation, the stem cell number was reduced $(P<0.05)$.

\section{DISCUSSION}

The neutrophil plays a central role in resistance to bacterial infection. In fact when a profoundly neutrophil depleted host develops bacterial sepsis, the likelihood of survival is small. This may be true even when the host possesses specific antibacterial antibody and is treated with appropriate antibiotics (10).

In order to guard against neutrophil depletion, the body maintains a large reserve of mature neutrophils, the neutrophil storage pool. In adult animals and man this reserve of PMN, band neutrophils and metamyelocytes contains 12-14 times the number of neutrophils in the circulating blood $(1,2,13)$. During bacterial infection these stored cells leave the marrow at an accelerated rate; even so, the reserve seldom becomes depleted. Presumably, depletion does not occur because the reserve is very large and also because it is constantly being replaced by the stem cells and neutrophil proliferative pool. In the present study we attempted to define the response of the neutrophil system to bacterial sepsis
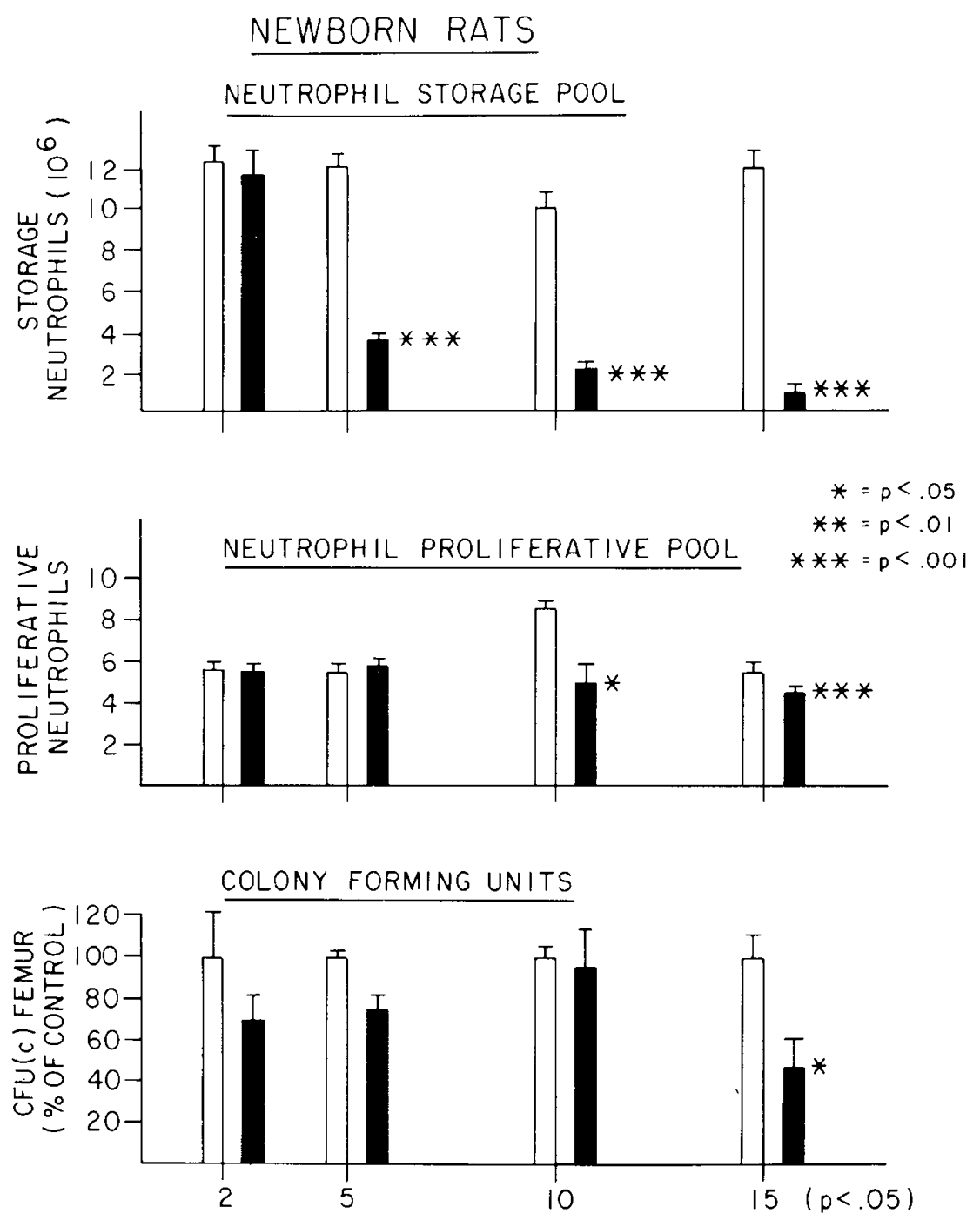

HOURS AFTER INOCULATION

Fig. 4. Storage neutrophils, proliferative neutrophils and unipotent stem cells $\left(\mathrm{CFU}_{\mathrm{c}}\right)$ in groups of infected versus control neonatal rats. 
by quantifying the unipotent granulocyte stem cells (CFU $)$, proliferative, storage and circulating neutrophil pools in groups of adult and neonatal animals.

The infected adult animals developed neutrophilia and a left shift. The neutrophils responsible for these blood changes obviously came from the NSP and quantification of this pool did indeed disclose a diminution $2 \mathrm{~h}$ following the inoculation. However, the reserve never did diminish to less than $60 \%$ of the control level. The neutrophil storage pool was repleted by $24 \mathrm{~h}$ and eventually increased to above control level as proliferative neutrophils matured. A 3-fold increase in stem cells $\left(\mathrm{CFU}_{\mathrm{c}}\right)$ occurred in the infected adults. The progeny of these stem cells presumably replace the neutrophils which mature from the proliferative into the storage pool. These studies demonstrate the reserve capacity and recovery capacity of the adult's neutrophil system. The myeloid tissue response of the neonatal rat to the same bacterial inoculum/g body weight was quite different from the adult. In the circulating blood, profound neutropenia accompanied a marked left shift. With these findings one might predict that the neonate's NSP had become depleted (5), and examination revealed that this did indeed occur. Furthermore, a reduction in proliferative neutrophil number and in $C F U_{c}$ was observed.

The inability of these neonates to respond to systemic infection with neutrophilia and maintenance of a large neutrophil reserve appears to represent a significant physiologic disadvantage. Further support for this hypothesis comes from the fact that replacement of neutrophils by granulocyte transfusion in septic, neutrophil depleted neonatal dogs improves survival (7), as does granulocyte transfusion in two populations of septic human neonates $(12,8)$. The mechanisms which are responsible for the different neutrophil responses of adults and neonates to bacterial infection are incompletely understood; however, we can point to a number of possibly relevant factors. First, the neonate simply possesses fewer neutrophils in the marrow neutrophil storage pool than does the adult $\left(0.12 \times 10^{8}\right.$ versus $4.0 \times 10^{8}$ per rat $)$ and even when expressed as stored neutrophils per $g$ of body weight, the neonate possesses only $25 \%$ of the adults reserve (9). Second, the storage pool of neonates appears to be more subject to neutrophil release stimuli, as shown in experiments in which stantardized neutrophil storage pool releasing stimuli were implanted into neonatal and adult animals (6). Third, the inability of neonatal neutrophils to efficiently migrate to inflammatory sites $(6,16)$ may further subject the neonate to an unrestricted stimulus for marrow neutrophil release which ultimately drains the marrow supply.

The inability of neonates to elevate their stem cell numbers during infection in a manner similar to the adult remains unexplained. From the current studies it is not clear whether this defect is one of an inherent inability of neonatal marrow proliferation, a nonspecific defect generated by shock during infection, or a direct toxic effect of products of uncontrolled infection on the marrow.

In conclusion, we administered $3 \times 10^{6}$ group $B$ streptococci/g body weight to groups of adult and neonatal rats and at intervals following the inoculum quantified stem cell, proliferative, storage and circulating neutrophils. The adults developed neutrophilia, a modest left shift, minimal NSP diminution and a 3-fold increase in stem cells, whereas the neonates developed neutropenia, a marked left shift, NSP depletion, and diminution of proliferative neutrophils and stem cells. Although the reasons for these differences are not entirely clear, this phenomenon of neutrophil depletion may be of critical importance in understanding the neonates' increased incidence of morbidity and mortality from sepsis.

\section{REFERENCES AND NOTES}

1. Cartwright, G. E., Athens, J. W.. and Wintrobe, M. M.: The kinetics of granulopoiesis in normal man. Blood, 24: 780 (1964).

2. Chervenick, P. A., Boggs, D. R., Marsh, J. C., Cartwright, G. E., and Wintrobe M. M.: Quantitative studies of blood and bone marrow neutrophils in normal mice. Am. J. Physiol., 215: 353 (1968).

3. Christensen, R. D. and Rothstein, G.: Exhaustion of mature marrow neutrophils in neonates with sepsis. J. Pediatr., 96: 316 (1981).

4. Christensen, R. D.. Shigeoka, A. O., Hill, H. R., and Rothstein, G.: Circulating and storage neutrophil changes in experimental type II group B streptococcal sepsis. Pediatr. Res., 14: 806 (1980)

5. Christensen, R. D.. Bradley, P. P., and Rothstein, G.: The leukocyte left shift in clinical and experimental neonatal sepsis. J. Pediatr., 98: 101 (1981).

6. Christensen, R. D. and Rothstein, G.: Efficiency of neutrophil migration in the neonate. Pediatr. Res., 14: 1147 (1980).

7. Christensen, R. D., Bradley, P. P., Priebat, D. A., Anstall, H. B., and Rothstein, G.: Granulocyte transfusions in septic canine neonates. Pediatr. Res., 16: 57 (1982)

8. Christensen, R. D., Rothstein, G., Bradley, P., Priebat, D., Bybee, B. and Anstall H. B.: Granulocyte transfusions increase survival in neonates with sepsis and neutrophil depletion. Pediatr. Res., (Abstract) 15:608 (1981).

9. Erdman, S. H., Christensen, R. D., Bradley, P. P., and Rothstein, G.: The supply and release of storage neutrophils: a developmental study. Biol. Neonate, 41 : 132 (1982).

10. Hill, H. R.: Phagocyte transfusion-ultimate therapy of neonatal disease? J. Pediatr., 98: 59 (1981)

11. Lancefield, R. C.: A micro-precipitin technique for classifying hemolytic streptococci and improved methods for producing antisera. Proc. Soc. Exp. Biol Med., 38: 473 (1938).

12. Laurenti, F., Ferro, R.. Isacchi, G. Panero, A., Savignoni, P. G., Malagnino, F., Palermo. D., Mandelli, F., and Bucci, G.: Polymorphonuclear leukocyte transfusion for the treatment of sepsis in the newborn infant. J. Pediatr., 98: 118 (1981)

13. Marsh, J. C., Boggs, D. R., Cartwright, G. E., and Wintrobe, M. M.: Neutrophil kinetics in acute infection. J. Clin. Invest., 46: 1943 (1967).

14. McFarland, W. and Libre, E. P.: Abnormal leukocyte response in alcoholism. Ann. Intern. Med., 59: 865 (1963).

15. Miller, M. E.: Host defenses in human neonate. Monographs in Neonatology. pp. 1-3 (Grune and Stratton, Inc., New York, NY, 1978).

16. Miller, M. E.: Chemotactic function in the human neonate: Humoral and cellular aspects. Pediatr. Res., 5: 487 (1971).

17. Naeye, R. R.: Underlying disorders responsible for the neonatal deaths associated with low apgar scores. Biol. Neonate, 35: 150 (1979).

18. Oshita, A. K. and Rothstein, G.: cGMP stimulation of granulocyte growth Blood, 49: 585 (1977).

19. Requests for reprints should be addressed to: Dr. Robert D. Christensen, Department of Pediatrics, University of Utah School of Medicine, 50 North Medical Drive, Salt Lake City, Utah 84132

20. This research was supported by a grant from the Thrasher Research Fund and by Public Health Service Grants HD-14419, Al-13I50 and AM-21140.

21. Received for publication August 27, 1981.

22. Accepted for publication November 16, 1981 . 